BULLETIN Bulletin hispanique

HISPANIQUE Université Michel de Montaigne Bordeaux

$119-2 \mid 2017$

La Égloga renacentista en el Reino de Nápoles

\title{
Le egloghe latine di Giano Anisio
}

"amico" napoletano di Garcilaso

Tobia R. Toscano

\section{(2) OpenEdition}

Journals

Edizione digitale

URL: http://journals.openedition.org/bulletinhispanique/5095

DOI: 10.4000/bulletinhispanique.5095

ISSN: 1775-3821

Editore

Presses universitaires de Bordeaux

\section{Edizione cartacea}

Data di pubblicazione: 1 décembre 2017

Paginazione: 495-516

ISBN: 979-10-300-0218-8

ISSN: 0007-4640

Notizia bibliografica digitale

Tobia R. Toscano, « Le egloghe latine di Giano Anisio », Bulletin hispanique [En ligne], 119-2 | 2017, mis

en ligne le 28 décembre 2020, consulté le 15 janvier 2021. URL : http://journals.openedition.org/

bulletinhispanique/5095; DOI : https://doi.org/10.4000/bulletinhispanique.5095 


\title{
Le egloghe latine di Giano Anisio, "amico" napoletano di Garcilaso
}

\author{
Tobia R. Toscano \\ Università degli Studi di Napoli Federico II
}

Dans sa première partie, cette contribution analyse les procédés utilisés par l'humaniste napolitain Giano Anisio dans son imitation de Virgile; dans la seconde, elle s'attache à l'interprétation des vers qu'il adresse à Garcilaso. Publiés en 1533, les deux poèmes de Giano Anisio à Garcilaso sont le premier témoignage de l'attention portée par le milieu des académies au poète espagnol et de son intégration rapide dans la "conversation» poétique de la capitale du Vice-Royaume.

Mots-clés: Giano Anisio, Garcilaso de la Vega, poésie latine, bucolique, Naples.

La presente contribución analiza el procedimiento adoptado por el humanista napolitano Giano Anisio en su imitación de Virgilio, deteniéndose en una segunda parte en la interpretación de los versos que este dirige a Garcilaso. Publicados ambos en 1533, los dos poemas de Giano Anisio son el primer testimonio de la atención de los ambientes académicos a la relación de Garcilaso y de su rápida inserción en la «conversación» poética de la capital del Reino.

Palabras clave: Giano Anisio, Garcilaso de la Vega, poesía latina, bucólica, Nápoles.

This paper focuses on the rhetorical and narrative patterns adopted by the Neapolitan humanist Giano Anisio in his imitation of Virgil and, more particularly, on the lines which he addressed to Garcilaso. The two poems by Anisio to Garcilaso (1533) are the first texts that show an academic interest towards the Spanish poet, who would then be "included" within subsequent debates on poetry by Neapolitan literary circles.

Keywords: Giano Anisio, Garcilaso de la Vega, latin poetry, pastoral literature, Naples. 
1. Giovanni Francesco Anisio, che in accademia latinizzò il suo nome in Giano, fu uno dei pontaniani più longevi: nato in un anno non precisabile dell'ultimo trentennio del ' $400^{1}$, risulta ancora tra i vivi nel 1539 , essendo morto probabilmente l'anno dopo ${ }^{2}$. Ebbe così modo, da giovane, di vivere la fase culminante della dinastia aragonese e assistere poi al suo tramonto e al mutamento istituzionale che fece del Regno di Napoli una dipendenza della corona di Spagna.

Come Pontano, sebbene non al suo livello, scrisse versi esclusivamente in latino pubblicando tutte le sue raccolte tra il 1531 e il 1538. Ma già da una testimonianza del 1539 sappiamo che i lettori di poesia avevano ormai una spiccata preferenza per la poesia in volgare, sicché non solo la sua opera, ma anche quella di Pontano, era giudicata un residuo del passato da condannare alla polvere degli archivi ${ }^{3}$. Il resto fece la penna velenosa di Nicolò Franco che riversò sul mite umanista l'accusa allora infamante di pedanteria e omosessualità ${ }^{4}$, sicché si spiega che l'insieme della sua opera solo negli ultimi anni abbia cominciato a sollecitare la curiosità dei lettor $\mathrm{i}^{5}$, sebbene siamo ancora lontani da una analisi sistematica che potrebbe probabilmente riservare qualche sorpresa.

La sua traiettoria biografica si muove tra il regno e la curia romana, anche se egli non fa mistero di preferire al trambusto della capitale l'otium tranquillo di cui poteva godere nel fondo avito di Domicella, ora in provincia di Avellino,

1. Per i riferimenti alla biografia e al corpus delle opere di Giano Anisio rinvio al bel contributo di Carlo Vecce, «Giano Anisio e l'umanesimo napoletano. Note sulle prime raccolte poetiche dell'Anisio», Critica letteraria, anno XXIII, no 88/89, 1995, pp. 63-80, che fa anche il punto sulla non copiosa bibliografia disponibile.

2. Sugli ultimi anni di Anisio, cf. T. R. Toscano, «Giano Anisio tra Nola e Napoli: amicizie, polemiche e dibattiti», in Id., L'enigma di Galeazzo di Tarsia. Altri studi sulla letteratura a Napoli nel Cinquecento, Loffredo, Napoli, 2004, pp. 79-102.

3. Giovanni Battista Pino, nel 1539, e quindi alla vigilia della morte di Anisio, presentando «Alli studiosi dela volgar lingua» il Ragionamento del terremoto, del nuovo monte, del aprimento di terra in Pozuolo nel anno 1538... di Pietro Giacomo da Toledo (Napoli, Giovanni Sultzbach, 22 gennaio 1539), insisteva sulla inutilità di attardarsi a coltivare la poesia in latino, visto che il pubblico ormai era pienamente conquistato dalla letteratura in volgare: «L'epigramme (come sapete) sono d'i minimi poemi che siano; nondimeno, chi epigrammista di tempi nostri ha giunto non che trapassato li Sali e l'argucie di Marciale e d'Ausonio? Certo, nullo. Hor, che speme si può haver nel resto? E, per confirmar il suo dire, portò per essempio il Pontano et l'Anisio, le cui scritture sono oltre che divinissime, degne d'eterno nuome et a pena si cognoscono dal mondo».

4. Sulla feroce satira di Nicolò Franco contro Anisio rinvio al mio contributo citato in nota 2. In una prospettiva più generale, si cf. Jesús Ponce Cárdenas, «Ma io son pure napolitano': Nicolò Franco e i circoli meridionali (1541-1543)», in Encarnación Sánchez García (dir.), Rinascimento meridionale: Napoli e il viceré Pedro de Toledo (1532-1553), Napoli, Tullio Pironti, 2016, pp. 203-234.

5. Segnalo due contributi di Sebastiano Valerio: «Il Protogonos di Aulo Giano Anisio: una tragedia nel tardo umanesimo napoletano», in S. Castellaneta e F.S. Minervini (eds.), Sacro elo profano nel teatro fra Rinascimento ed Età dei lumi, atti del Convegno di Studi (Bari, 7-10 febbraio 2007), Bari, Cacucci, 2009, pp. $39-56$ e «Il De principe di Aulo Giano Anisio», in Acta Conventus Neo-Latini upsalensis: Proceedings of the XIV International Congress of Neo-Latin Studies (Uppsala 2009), London-Leiden, Brill, 2012, pp. 1165-1175. 
sul versante orientale del monte Sarno, nel cui territorio si trovava (e ora ne avanza qualche resto) l'abbazia di Santa Maria delle Grazie, il cui beneficio gli era stato conferito nel 1513 dal cardinale Luigi d'Aragona ${ }^{6}$. Domicella viene così trasfigurata in tanti carmi nella ninfa Domicilla abbellita e vezzeggiata dal suo patronus in modo da poter gareggiare con ninfe ben più prestigiose come Antiniana o come Mergilina cantate da Pontano e Sannazaro ${ }^{7}$.

Il nostro Anisio volle chiamarsi Giano, e come Giano egli appare bifronte, nel senso che la sua vasta produzione, se si scorrono le prime due raccolte pubblicate nel 1531 e nel 1532, guarda al passato anche remoto della giovinezza, sotto il segno di un rapporto di clientela con il cardinale Pompeo Colonna, morto appunto nel 1532, quindi costringendo Anisio negli anni seguenti, dal liber del 1533 a quello del 1538, a costruire una nuova rete di relazioni, che include in primis il viceré don Pedro de Toledo, giunto a Napoli nel 1532: fanno spicco in questa fitta trama i versi indirizzati ai nuovi venuti quali Garcilaso de la Vega e Juan de Valdés. Ma di questo si dirà più avanti. Negli ultimi anni di vita egli appare sempre più legato ad altro personaggio importante della corte vicereale, Bernardino Martirano, segretario del Regno, e al fratello di lui Coriolano, che ospitavano gli amici letterati nella villa suburbana di Leucopetra, resa ancor più prestigiosa dal soggiorno di Carlo V reduce dall'impresa di Tunisi nel novembre del $1535^{\circ}$.

6. In una allegazione forense di Carlo Gagliardi (senza note tipografiche, ma quasi sicuramente stampata a Napoli e sottoscritta e datata, a p. 140, Napoli 10 ottobre 1766) intitolata Per la spedizione del regio exequatur sulla pontificia Bolla collativa della Chiesa parrocchial di Domicella [...] contra l'impedimento opposto [...] dal Monastero di S. Lorenzo d'Aversa... (Biblioteca Provinciale di Avellino, Bibl. di G. e S. Capone P inv. 1360) è riportata la trascrizione della concessione del beneficio a Giovanni Francesco Anisio da parte del cardinale Luigi d'Aragona "Datum Romae 27 novembris 1513".

7. Molti anni dopo la morte di Anisio si ricorderà di lui e di Domicilla Antonio Minturno, ospite e forse allievo di Giano ai tempi della peste del 1526, che includerà nei suoi Poemata ad M. Antonium Columnam (Venetiis, Apud Io. Andream Valvassorem, 1564, cc. 19r-21r) una lunga elegia intitolata Ad Ioannem Franciscum Anisium. Domicilla, in cui dopo un lungo discorso sulla peste che non risparmia niente e nessuno, ricorda nostalgicamente «Domicilla bonis sedes dilecta Camenis», fino all'apostrofe finale: "Fortunate senex, cui tam iucunda tenere / rura datum leteque locis gaudere beatis, / sic Dea, quae rebus solet invidisse secundis, / sit bona, sit felix, optata tibi ocia servet» ( $V$ ecchio fortunato, cui fu concesso abitare campi così fertili e godere in letizia luoghi tranquilli, così la dea di solito invidiosa della felicità sia clemente, sia prospera, e ti conservi il desiderato riposo»). Il tardivo omaggio di Minturno è tanto più rilevante se si tiene conto della selezionatissima platea dei destinatari dei suoi carmi latini: Imperatore, papi, grandi funzionari dell'impero, viceré, vescovi. Gli unici letterati sono Paolo Giovio e Giano Anisio, che viene ricordato, per quanto assente al dibattito, da Lucio Vopisco nel De poeta dello stesso Minturno (Venezia, Rampazzetto, 1559, libro III, p. 178) come il letterato a quel tempo (circa 1526) meglio attrezzato per discutere di teatro tragico. Anisio avrebbe pubblicato nel 1536 la tragedia Protogonos (Napoli, Sultzbach).

8. Ho provato a ricostruire la rete di relazioni dei fratelli Martirano in miei precedenti lavori: Bernardino Martirano, Il pianto d'Aretusa, ed. T. R. Toscano, Napoli, Loffredo, 1993 e, da ultimo, T. R. Toscano, «Un nobile cosentino al servizio dell'Impero: otia e negotia di Bernardino Martirano tra eredità pontaniana e sperimentalismo in volgare», in D. Gagliardi (ed.), La cultura ispanica nella Calabria del Cinque-Seicento. Letteratura, Storia, Arte, Soveria 
2. Venendo al tema specifico, mi pare sia del tutto scontato immaginare che, scrivendo versi latini, un umanista utilizzi lessico, stilemi e costrutti degli auctores sulle cui opere ha appreso la grammatica. Non diversamente dai contemporanei cultori della poesia in volgare, che in maniera progressivamente esclusiva modellarono i loro versi sulle rime di Petrarca, senza abbandonare suggestioni di altri autori e dei classici latini e dei poeti neolatini a loro contigui, anche Anisio esibisce la sua galleria di auctores la cui fungibilità va rapportata al genere trattato. Pertanto non desta stupore che nel contesto dell'invocazione a Talia in apertura dei Varia poemata (Neapoli, I. Sultzbach, 1531), presentando il suo libellus lo affidi alla protezione di Catullo e di Orazio dei quali si dichiara alunno:

\section{Hoc modo tibi nuncupo, Catulle, Horati et tibi quicquid est libelli, quando vos habui mihi magistros \\ (c. $2 \mathrm{r}){ }^{9}$}

Questa dichiarazione un po' contrasta con la mole del libellus e con la pluralità dei generi trattati, giacché scorrendo le 320 pagine che lo compongono si ha modo di incontrare sei egloghe in esametri, che non formano una sezione a parte, ma sono frammiste a componimenti in altri metri e su temi diversi. Questa apparente confusione è preventivamente segnalata dal breve saluto $A d$ lectorem (c. $2 r$ ) ricordandosi che i carmi sono stampati rispettando la cronologia della loro composizione:

\section{Eo ipso ordine quo mihi exciderunt ore carmina continet libellus. Casta id simplicitas mihi imperavit. ${ }^{10}$}

Un'idea di casta simplicitas coniugata alla varietas indicata nel titolo, quasi per suggerire l'idea di raccolte costruite di fragmenta, ai quali affidare le tracce del proprio vissuto.

Tale criterio cronologico appare osservato anche nella successione delle sei egloghe, un numero che potrebbe voler indicare una misura aurea, una sorta di invalicabile soglia, tenendo a mente che sei sono le egloghe di Pontano e che se le Piscatoriae di Sannazaro sono cinque si può tener conto che esse fanno corpo con i Salices, stampati nella princeps del 1526 immediatamente dopo le egloghe, contigui per l'ambientazione acquatica (dal golfo di Napoli alle correnti del fiume Sarno). Non sono in grado di dire se la scelta abbia un significato preciso. Certo è che rispetto al modello virgiliano Anisio sembra voler programmaticamente far propria l'opzione di un corpus più ridotto seguendo l'esempio di Pontano e Sannazaro. Ciò implica che leggendo la poesia

Mannelli, Rubbettino, 2013, pp. 115-28.

9. «Pertanto a te, Catullo, e a te, Orazio, consacro, qualunque sia, il mio libercolo, poiché vi ho avuti come guida».

10. «Il libretto dispone i carmi nell'ordine medesimo in cui furono pronunciati. Ciò mi comandò la casta semplicità». 
bucolica di Anisio si debba tener conto non solo delle scoperte suggestioni e citazioni di fonte virgiliana e di altri autori latini, ma anche guardare al fitto reticolo di allusioni e rinvii alla produzione dei due "maestri" napoletani, ai quali il più giovane Anisio guardò sempre con deferenza.

Non per caso infatti la prima egloga si intitola Melisaeus ${ }^{11}$, in cui Micone ricanta a Egilo la naenia eseguita da Giovanni Cotta sulla tomba di Pontano nove giorni dopo la sua sepoltura: una volta tanto si riescono agevolmente a cogliere le allusioni disseminate nel testo e a individuare i personaggi che si nascondono dietro i nomi pastorali. Meliseo è il nome che Pontano aveva scelto per sé nella seconda delle sue eglogae in cui piange la morte della moglie Adriana; Micone, custode di greggi, si comprende dal contesto essere Anisio stesso, mentre Egilo, padrone del gregge lasciato incustodito, è Pompeo Colonna. Per l'identificazione di Egilo con Pompeo Colonna ci viene in aiuto Anisio stesso in un breve carme falecio indirizzato a Ad Pompeium Columnam (c. $11 r$ ) che si legge subito dopo l'elegia:

\title{
Pompei, quando ego nil non tibi debeo seu pascam pecus in maenaliis iugis, te sylvae resonent et nemora Aegilon, seu laeta arva canam $[\ldots] .{ }^{12}$
}

La collocazione temporale è precisa: Pontano era morto il 17 settembre 1503, e di Giovanni Cotta si dice che

\author{
[...] nuper qui his appulit oris, \\ (non nosti, non fama tuas pervenit ad aures?) \\ viseret ut viridis Meliseum in flore senectae,
}

11. Una puntuale lettura di questa egloga è offerta da C. Vecce, «L’egloga Melisaeus di Giano Anisio tra Pontano e Sannazaro", in S. Carrai (ed.), La poesia pastorale nel Rinascimento, Padova, Antenore, 1998, pp. 213-34.

12. «Poiché ti devo tutto, o Pompeo, sia che io pascoli il gregge sui colli arcadici, le selve e i boschi ti ricantino sotto il nome di Egilo, sia che io canti i fertili campi...». Vecce (ivi, p. 230) argomenta che al momento della composizione (1503) dell'egloga dietro il pastore Egilo si celasse Egidio da Viterbo e che l'epigramma sia «un'operazione tardiva ed encomiastica, che oblitera il patrono originario di Anisio». Credo però che non si possa negare l'incombente presenza di Pompeo Colonna in tutto il liber anisiano, fin dalle carte iniziali dove si legge (cc. 4-5) un lungo carme De natali Pompeii Columnae, in cui Anisio dichiara di essere un suo cliente: «Cras natalis erit mei patroni / Pompeii, patris elegantiarum». Aggiungerei che il riferimento alla paupertas del dominus ha la sua pregnanza (cf. vv. 129-31 del testo riedito da Vecce, p. 234: «[...] Te munere pauper / Aegilus exiguo donat. Sed si mibi detur / speratas ut oves Tyberina ad flumina pascam, / mecum eris...»), se riferita alla condizione personale di Pompeo Colonna al momento della morte di Pontano. Era infatti ancora in vita papa Alessandro VI, che il 20 dicembre 1501 aveva scomunicato e privato dei beni Pompeo insieme ad altri 11 membri della famiglia Colonna, costringendolo a fuggire a Napoli. I Colonna furono reintegrati nei loro possedimenti romani da Giulio II, eletto il $1^{\circ}$ novembre 1503, e quindi Pompeo Colonna avrebbe avuto modo di tornare "ricco" Tyberina ad flumina e così onorare la promessa fatta a Micone-Anisio (per la biografia di Pompeo Colonna si rinvia alla "voce" di F. Petrucci per il Dizionario biografico degli italiani, Roma, Istituto dell'Enciclopedia italiana, 1982, t. 27). 
La scelta di dare principio al proprio esercizio bucolico muovendo dall'apoteosi di Pontano appena defunto, al pari del Dafni virgiliano tratteggiato come benefico nume tutelare dei pastori, sembra dettata da una forma di rispetto verso il venerato maestro: una sorta di ideale passaggio di consegne alla generazione più giovane, in un tempo in cui Sannazaro era ancora in Francia. Il Regno di Napoli era allora dilaniato dalla guerra franco-spagnola che, nel dicembre di quello stesso 1503, viene risolta dall'acume strategico congiunto del Gran Capitano e Bartolomeo d'Alviano, che fanno passare il loro esercito sulla sponda nord del fiume Volturno controllata dai francesi: la seconda egloga è dedicata alla vittoria delle armi spagnole e registra lo stupore per lo stratagemma escogitato dagli spagnoli di costruire un ponte di barche per trasbordare l'esercito sull'altra sponda:

\section{[...] Consalvus io fortissimus heros pontibus est ausus tumidum transmittere Lyrin, nec numerosa manus gallorum sustinet hostem, sed conversa fugam in foedam dat terga secanda invictae Hesperiae insigni cum clade phalangi [...]} (c. $12 \mathrm{r}){ }^{14}$

È di qualche interesse notare che questa seconda egloga, intitolata Murenus (c. $11 \mathrm{v}$ ) sembra una mescidanza del genere bucolico e piscatorio, in quanto gli interlocutori sono un "Murenus piscator», insieme a "Quercius et Hircanus pastores", con un altro personaggio di nome Cursor, che reca notizie dal teatro di guerra. Confesso di non essere in grado di identificare nessuno degli interlocutori che gareggiano nella lode del Gran Capitano promettendo di innalzare trofei in suo onore.

Né meno problematico è squarciare il velo dell'allegoria in cui è involta la terza egloga intitolata Sapientia (cc. 43-44), mentre la quarta pone in scena direttamente Sannazaro-Syncerus, tornato dall'esilio, e un pastore di nome Aepolus, da intendersi come alter ego di Anisio. Siamo quindi nel 1505. L'ambientazione è un paesaggio montano lontano dalla città. Questa egloga è per Anisio anche un pretesto per esibire l'amicizia di antica data con Sannazaro ("Quod salvus, Syncoere, tuos patriamque revisis / gaudeo et antiquum video atque amplector amicum», c. $45 v)^{15}$, che nelle prime battute del colloquio aveva

13. «... colui che da poco approdò ai nostri lidi (non lo conosci, non ti pervenne alle orecchie la notizia?) per vedere Meliseo nel fiore di una vigorosa vecchiaia, sostegno dei pastori, e di lui quanta stima faceva Meliseo e quante volte disse: "Cotta è un'altra speranza di Febo"».

14. «[...] Viva! Consalvo invincibile eroe ebbe l'ardire di scavalcare il Liri ondoso con ponti (di barche) né la numerosa schiera dei Galli regge il nemico, ma volta in vergognosa fuga offre la schiena da trafiggere alla invitta falange di Esperia con un strage che resterà famosa».

15. «Sono felice, Sincero, che incolume ritorni ai tuoi e alla patria, e io posso vedere e abbracciare l'amico di antica data». 
chiesto i motivi che lo avevano indotto ad abbandonare l'esercizio poetico ("Aepole sic tacitus solis in montibus erras / cum grege subtristi; quondam sub Daphnide nostro / et doctis musis et Apolline digna canebas", c. 45r). È l'assenza di Dafni ad aver determinato lo sconvolgimento dell'ordine naturale:

Omnia sunt eversa, ipsa et fidissima mater
fert tristem loeto ac fallacem in semine frugem.
In taxum felix mutata est Palladis arbos,
nobilis et Bacchi in vaccinia vitis amara,
induit helleborum tristem cytisusque thymusque,
usque adeo fracta est aetas effoetaque tellus! ${ }^{16}$
Quid faciam, Syncoere? hominum commercia vito (c. 46r). ${ }^{17}$

È uno dei pochi casi in cui l'autore ha ritenuto di dover dichiarare, nel breve elenco di obiter adnotata posto alla fine del volume, l'identità del personaggio: «Sub Daphnidis persona signatur Federicus rex Aragonius». E il moderno Dafni è dotato dei medesimi benefici poteri del Dafni virgiliano:

Quid Daphnis faciebat? pastorum arbiter, ille
hic erat assuetus morbos arcere malignos
aut campi aut pecoris, mala si contagia forte
inciderant doctaque manu disponere vites
atque fera inserere externos super arbore foetus. ${ }^{18}$

A differenza del Dafni virgiliano però al re Federico è toccata la sorte di morire lontano dalla patria senza ricevere i meritati onori funebri («Pro superi! haec meruit crudelia funera Daphnis?»).

Le egloghe rimanenti trattano temi più lieti e sono distanziate cronologicamente dalle precedenti: l'ultima è di ambientazione romana e il titolo Coritius mette in scena Johann Goritz (Ianus Coritius), prelato lussemburghese e figura importante della Curia romana e mecenate, nonché animatore di un circolo accademico con il quale ebbe qualche consuetudine Giano Anisio negli anni trascorsi a Roma al seguito di Pompeo Colonna, come testimoniano i due epigrammi conferiti ai Coryciana, raccolta di poesie latine pubblicata a Roma nel $1524^{19}$. Coritius invita Foenius e Peridon a una gara di

16. Il verso riprende con lieve variazione Lucrezio, De rerum natura 2, 1150: «Iamque adeo fracta est aetas effetaque tellus». Le frequenti riprese da Lucrezio, oltre che da Ovidio e anche dagli antichi poeti cristiani sono «segno che la poesia di Anisio nasceva ancora sotto l'influenza della vecchia scuola quattrocentesca» (Vecce, «L’egloga Melisaeus di Giano Anisio tra Pontano e Sannazaro", cit., p. 222).

17. «Tutte le cose sono in confusione: e persino la madre terra produce triste e ingannevole messe dal seme buono. L'ulivo è mutato in tasso e la nobile vite di Bacco in giacinto amarulento, e il citiso e il timo si rivestirono di elleboro nero, a tal punto è sconvolta e infiacchita la terra! Che fare, Sincero? Evito il consorzio umano».

18. «Cosa faceva Dafni? Guida dei pastori, qui egli era solito tenere lontano perniciose infezioni o dei campi o delle greggi, nel caso si fossero propagati pericolosi contagi, e allineare le viti con mano esperta e innestare virgulti nuovi su alberi selvatici».

19. Effettivamente nella raccolta sono compresi (a cc. I $4 r$ e L1 $r$ ) due componimenti attribuiti 
canto (c. 75v) mettendo in palio una "pateram... Myronis / prisci opus». L'egloga riproduce lo schema della bucolica settima di Virgilio e il fatto che Coritius dichiari vincitore Peridone ci fa sospettare che possa trattarsi di Anisio.

3. Di particolare interesse l'egloga quinta, intitolata Ursus: la vicenda è ambientata nel mese di maggio, al tempo dei pellegrinaggi che i pastori compiono al santuario mariano di Montevergine, per chiedere protezione sui campi e sulle greggi. È un lungo racconto di 124 esametri affidato alla voce di Micone-Anisio: lasciate le greggi «Sebethi ad pascua laeta», si incammina in compagnia di Ianus, del quale nessuno era più abile «ducere seu choreas seu doctos dicere versus» (c. 73r). Vecce ha proposto di identificare Ianus con Aulo Giano Parrasio, e l'ipotesi sarebbe sicuramente affascinante, se non urtasse in qualche difficoltà di cronologia e con il particolare non meno importante che Parrasio, grande filologo classico, non pare abbia lasciato tracce di attività poetica. Lasciata Napoli, scorgono all'orizzonte il Vesuvio dai pendii bruciati dalla lava ("Interea ambesa surrexit fronte Vesevus», c. 73v), le cui tremende eruzioni avevano costretto gli antichi coloni di Partenope e di Cuma a migrare nel territorio nolano. Si vedevano ormai in lontananza i «nolana sepulchra» ${ }^{20}$, quando irrompe sulla scena a gran voce il giovane Ursus, che a questo punto del racconto è possibile identificare con Enrico Orsini, ultimo conte di Nola ${ }^{21}$, al quale Micone-Anisio aveva insegnato l'arte poetica ("Ursus erat iuvenis prisca de gente deorum / quem puerum docui calamos inflare canoros»). Il discepolo confida al maestro che ormai la sua unica cura è Merine, Maria Sanseverino, prossima ormai a diventare sua sposa («Care Mycon optatus ades nostris hymenaeis», c. 74r). Questo riferimento induce a collocare l'egloga al 1513, anno delle nozze del conte di Nola ${ }^{22}$. Mentre si concedono uno spuntino, appare sulla scena un nuovo personaggio indicato come «Sarnius heros», dagli inconfondibili connotati nobiliari («equo celsus, redimitus tempora quercu»), e che potrebbe essere un membro della famiglia Tuttavilla, conti di Sarno, il

a Io. Franciscus Anysius il primo e a F. Anysius il secondo. Tuttavia il primo dei due (Esse subiratus Cipelli videris, amici) va restituito a Cosma, fratello di Giano, perché si trova ristampato nel volume dei suoi Poemata (Neapoli, I. Sultzbach, 1533, c. 28r, con il titolo Ad Cipellum).

20. Sono tombe di epoca romana poste sulla strada da Napoli a Nola, quasi emblema dell'antichità nolana, gli «antiqui tumuli» che pure Ambrogio Leone aveva voluto far riprodurre da Girolamo Moceto in una delle tavole che corredano il De Nola (Venezia 1514).

21. Enrico Orsini, figlio di Gentile e di Caterina d'Aragona, ottenne la contea di Nola nel 1509 per donazione del nonno Nicolò, conte di Pitigliano e condottiero in quegli anni al servizio di Venezia. L'investitura fu confermata da Ferdinando il Cattolico nel 1510. Dal matrimonio con Maria Sanseverino, figlia di Bernardino principe di Bisignano, non nacquero eredi e la signoria nolana degli Orsini ebbe il colpo di grazia in occasione dell'invasione di Lautrec nel 1528, in quanto Enrico fu dichiarato ribelle per aver seguito la parte francese e sarebbe stato giustiziato se la morte, avvenuta nel 1533, non lo avesse sottratto al boia: cf. G. Vincenti, $L a$ contea di Nola dal sec. XIII al XVI. Ricerche storiche e feudali, Napoli, Girolamo Coppini, 1897, pp. 56 e $59-60$.

22. Cf. P. Manzi, La reggia degli Orsini di Nola (1470-1970), Roma, Istituto storico e di cultura dell'Arma del genio, 1971, p. 45. I capitoli matrimoniali furono sottoscritti a Napoli il 28 giugno 1513: G. Vincenti, La contea di Nola dal sec. XIII al XVI, cit., p. 58. 
quale invita Micone a ripetere il canto che gli aveva consentito di superare Licida, venuto a gareggiare dai monti degli Osci.

Micone canta l'infelice amore di Polifemo per la ninfa Galatea, che riscuote l'applauso unanime degli astanti. Al termine Ursus chiede notizie degli amori di Cabanilio. Micone riferisce le pene d'amore di questi per Dori. L'egloga si conclude, con modalità tipica della poesia pastorale, con l'invito di Ursus agli ospiti a trascorrere insieme la parte restante della notte, per riprendere il cammino il giorno seguente.

Il componimento presenta una struttura alquanto complessa: entro la cornice narrativa della sosta a Nola, ospiti di Enrico Orsini, si sviluppa la rievocazione di una gara poetica tra Micone e Licida, seguita a sua volta dal lamento di Cabanilio per l'ingrata Dori. I temi e i personaggi riconducono inevitabilmente a Sannazaro e al suo milieu. Il nome pastorale adottato da Anisio è Micone, che ha lo stesso nome dell'interlocutore di Licida nella prima piscatoria di Sannazaro. L'infelice amore di Licone per Galatea è argomento trattato da Sannazaro nella seconda egloga; il Cabanilius di Anisio che si querela di Dori è il medesimo Traiano Cavaniglia, conte di Montella e di Troia, cui Sannazaro dedica i Salices, che si leggono dopo la quinta delle piscatoriae. Ne dovrebbe conseguire che Micone-Anisio un tempo aveva gareggiato con Sannazaro sul tema degli amori di Galatea, riportandone vittoria, almeno stando a quanto si fa dire dal «Sarnius heros». Sembra una pretesa francamente un po' eccessiva. Ma questo elemento però riconduce a una fase della vita in cui i due poeti potrebbero essere stati insieme ospiti nel feudo dei Cavaniglia a Montella, nello stesso periodo in cui vi fu anche Giovanni Cotta, autore a sua volta di versi indirizzati ai fratelli Anisio e al fiume Calore che nasce in territorio di Montella. Ricordo, con beneficio d'inventario, che nelle annotazioni alla Vita di Sannazaro scritta da G. B. Crispo (ed. Venezia 1725, p. XVI) si riferisce che «in memoria di tal dimora in quei luoghi del Sanazzaro, il Conte fece dal nobile pennello di Andrea Sabbatino, detto di Salerno, dipingerlo al vivo in un quadro dell'Assunta, in luogo di un degli Apostoli al sagro tumulo, che nel territorio di Montella, nella chiesa de' Felloni [Folloni], de' Frati Francescani, si osserva, insieme con quella del Cotta e dell'Anisio».

4. La testura dell'egloga è tramata di riprese dal corpus virgiliano, soprattutto dalle Bucoliche, ma con significativi apporti anche dalle Georgiche e dall'Eneide. Accanto a Virgilio si risente la presenza di altri autori, Lucrezio e Ovidio in primis, che agiscono nella memoria di Anisio a vari livelli, dal semplice reimpiego di un lemma abbastanza raro al recupero di interi emistichi, ma soprattutto nelle clausole pentasillabiche dell'esametro.

Uno specimen abbastanza completo di questa articolata procedura di prelievi si può osservare nei vv. 112-15 che concludono il lamento d'amore di Cabanilio (c. $75 v$ ): 
nec liquido aestivae cantabunt ore cicadae, quam, mea lux, moriens tanti sim oblitus amoris. ${ }^{23}$

Il primo verso ricombina due versi del canonico adynaton dell'egloga prima di Virgilio (59-60: "Ante leves ergo pascentur in aethere cervi / et freta destituent nudos in litore piscis») conservandone la filigrana sonora, pur nella diversa distribuzione dei costituenti, recuperando più avanti la clausola gutture corvi da Georgiche 1, 423 («et laetae pecudes et ovantes gutture corvi») con ulteriore prelievo di cellula ritmica in fine del verso seguente (ecl. 5, 77: «dumque thymo pascentur apes, dum rore cicadae»), sia pure modificata nel significato, con l'aggiunta dell'agg. aestivae riferito a cicadae suggerito probabilmente da Ovidio, Ars 1, 271 ("Vere prius volucres taceant, aestate cicadae»).

Altro caso di imitazione a intarsio ai vv. 72-78, dove si può osservare che il lamento di Polifemo per l'ostinato rifiuto di Galatea riadibisce alcune tessere dal canto di Coridone per Alessi della seconda egloga virgiliana, con ulteriori prelievi da altre opere:

O quoties Galatea tibi servavimus hoedos: ${ }^{24}$
creverunt hoedi, crevit mihi flamma medullis. ${ }^{25}$
An quia sim pauper spernis me, nympha? quis unquam ${ }^{26}$
tam dives pecoris, tam pingui casei abundans? ${ }^{27}$
An quia sim turpis? Nuper me in littore vidi, ${ }^{28}$
dum mare marmoreum turbarent flamina nulla. ${ }^{29}$

Anche l'aggettivo marmoreum riferito a mare è di provenienza virgiliana (Aen. 6, 729: «et quae marmoreo fert monstra sub aequore pontus»).

Un caso di combinazione in versi contigui di secondo emistichio ripreso ad verbum con rincalzo di clausola pentasillabica nel verso seguente si osserva ai vv. 5-6:

Ianus erat mecum, quo non praestantior alter ducere seu choreas seu doctos dicere versus

da confrontarsi con

Misenum Aeoliden, quo non praestantior alter / aere ciere viros...

(Verg. Aen. 6, 164-5)

23. «Ma non nasceranno altri pesci nel mare, né gli antri riecheggianti risponderanno ai miei lamenti, né canteranno con la gola arrochita i corvi d’inverno, né d'estate le cicale friniranno con limpida voce, prima che io, mia luce, morendo mi dimentichi di amore così intenso».

24. Verg. ecl. 5, 12: «Incipe, pascentis servabit Tityrus haedos».

25. Verg. georg. 3, 271: "continuoque avidis ubi subdita flamma medullis».

26. Verg. ecl. 3, 74: "Quid prodest quod me ipso animo non spernis, Amynta».

27. Verg. ecl. 2, 20: "Quam dives pecoris, nivei quam lactis abundans».

28. Verg. ecl. 2, 25: "Nec sum adeo informis: nuper me in litore vidi».

29. Concettualmente analogo a Verg. ecl. 2, 26: «cum placidum ventis staret mare...». "Quante volte, Galatea, ho conservato dei capretti per te: sono cresciuti i capretti, mi è cresciuta la passione nelle midolla! Forse mi disprezzi, o ninfa, perché sono povero? Chi mai (come me) così ricco di gregge, così fornito di grasso formaggio? O forse perché sono sgraziato? Poco fa mi sono specchiato sulla spiaggia mentre brezza alcuna increspava il mare liscio come un marmo». 
Qui anche si nota come il reimpiego dell'emistichio riferito a Miseno induca il dattilo ducere a inizio verso per suggestione del dattilo aere in posizione analoga nel luogo virgiliano, prima di planare su lessico e contesto più spiccatamente bucolici di $e c l .5,2$, verso topico il cui primo emistichio sarà ripreso al successivo v. 27 («quem puerum docui calamos inflare canoros»).

Altri casi di ripresa di intero emistichio a v. 41 ("fertur equo celsus redimitus tempora quercu» da georg. 1, 349: «quam Cereri torta redimitus tempora quercu») o con lieve adattamento a v. 48 ("Hic ego, nam hirsuto pendebat fistula collo» da ecl. 7, 24: "Hic arguta sacra pendebit fistula pinu»).

Più nutrita la serie di riprese in clausola, che possono essere vere e proprie citazioni, come ai vv. 2 («maio mense solent pastores vota quotannis»: ecl. 5, 79: "Ut Baccho Cererique, tibi sic vota quotannis») e 42 ("Omnia pastorum, nympharumque omnia plena»: ecl. 3, 60: «Ab Iove principium musae: Iovis omnia plena» ${ }^{30}$; o recuperi con lievi variazioni di costrutto necessitate dal diverso contesto (il v. 16: "usque a Parthenope et Cumis migrasse colonos» riecheggia ecl. 9, 4: "diceret: "haec mea sunt; veteres migrate coloni"» e il v. 47: "huc venit cantu et calamis certare paratus» riadatta Aen. 5, 108: «Visuri Aeneadas, pars et certare parati») ${ }^{31}$.

5. Venendo al rapporto tra Giano Anisio e Garcilaso de la Vega, occorrerà innanzitutto sgombrare il campo dalla piccola svista occorsa a Eugenio Mele ${ }^{32}$, e attraverso di lui transitata nella tradizione dei commenti garcilasiani, che attribuì a Cosma Anisio, fratello di Giano, i due brevi carmi indirizzati a Garcilaso. L'equivoco si spiega perché nel 1533 furono stampati i Poemata di Cosma (Neapoli, I. Sultzbach), che raccolgono la varia produzione del fratello minore del Nostro, medico di professione e anch'egli al servizio del cardinale Colonna. Tale volume presenta una particolarita ${ }^{33}$ sfuggita ai lettori: dopo il frontespizio principale, seguito dai primi quattro libri, tra le cc. $76 r$ e $100 r$, è inserito un Variorum poematum liber di Giano Anisio con nuovo frontespizio interno e in questo liber di Giano si leggono i versi a Garcilaso, e precisamente

30. La clausola omnia plena presenta 4 occorrenze anche in Lucrezio.

31. Il campione proposto è di necessità limitato. Sarebbe tuttavia utile pubblicare il testo integrale dell'egloga di Anisio corredata dai rinvii a Virgilio e agli altri autori classici, senza trascurare le suggestioni provenienti, soprattutto per l'episodio di Galatea, da Sannazaro e forse più da Pontano, che due volte nella Lyra e poi ancora in una saffica dell'Antonius rimodula il canto di Polifemo per la ninfa ritrosa.

32. "Las poesías latinas de Garcilaso de la Vega y su permanencia en Italia», Bulletin Hispanique, vol. 25, 1923, pp. 108-48, a pp. 141-142.

33. Segnalata da me nel 1988, allorché estrassi da questo liber "disperso" il precoce distico in morte di Ludovico Ariosto: T. R. Toscano, «Due "allievi” di Vittoria Colonna: Luigi Tansillo e Alfonso d'Avalos", in Critica letteraria, anno XVI, no 61, 1988, pp. 739-773 (a pp. 764-765, nota 69). Manca infatti nel catalogo ricostruito da Carlo Vecce, «Giano Anisio e l'umanesimo napoletano. Note sulle prime raccolte poetiche dell'Anisio», cit. e di qui riproposto da Micaela Ricci in Aulo Giano Anisio, Melisaeus, Foggia, Edizioni del Rosone, 2008, pp. 32 e 65. 
alle cc. $91 v$ e $94 r$. È utile rileggerli se non altro perché sono la più precoce testimonianza letteraria sulla presenza di Garcilaso a Napoli e in assoluto contengono uno dei primi giudizi pubblici sull'opera e forse sulla persona e sul carattere del poeta spagnolo.

Ciò che a me pare degno di particolare attenzione è la tonalità colloquiale dei versi, che lascia scorgere nelle filigrane del discorso un rapporto già consolidato nei pochi mesi dopo l'arrivo a Napoli, che, se non implica un rapporto di amicizia (considerato il silenzio di Garcilaso su di lui nell'opera superstite), certo autorizza a ipotizzare una comune frequentazione nel contesto più ampio della società aristocratica napoletana nei mesi in cui venivano stabilendosi i primi legami con l'entourage di don Pedro de Toledo, arrivato a Napoli il 4 settembre del 1532. E Garcilaso era certamente persona che non passava inosservata.

Nel primo carme in endecasillabi faleci Anisio contrappone la vita serena che può trascorrere nella villa di Domicella alla tirannia del lusso e alle ansie della vita cittadina:

\author{
Ad Charisylam seu Charsilassum \\ Charisyla amice, divitis proles Tagi, \\ cur invides mihi otium pulcherrimum, \\ hos et recessus perbeatos liberos \\ vestris ab istis regibusque et luxibus? \\ Hic integra vel fraxino imposta focis \\ ardente Mulcibre frigus urens pellimus, \\ vernis cachinno dulce perstrepentibus. \\ Hic Sirius cum saevus urit aequora \\ flavis aristis compta fluctuans caput, \\ proiecta ramos fagus alta umbraculis \\ aut vitis imbricata scaena plurima \\ arcet calorem delicato frigore, \\ felix opaco tegmine atque Liberi \\ laetis racemis suavisucco nectare. \\ Non ora centum docta voxque ferrea \\ praeconio aequent ruris aurei bona \\ quis cum vel urbis ista mutes gaudia \\ contacta et hydrae viro et ore Cerberi. ${ }^{34}$
}

34. "Caro Garcilaso, prole del ricco Tago, perché mi neghi il piacere di un bellissimo ozio: e di questo ritiro assai felice senza le tirannie di codesti vostri lussi? Qui si mette al fuoco anche un intero frassino e con Vulcano ardente si scaccia il freddo bruciante; la servitù intanto con piacere schiamazza e ride. Qui quando Sirio spietato agitando il capo brucia le distese adorne di bionde spighe, quando l'alto faggio protende i rami a pergolati o il paesaggio della foltissima vite embriciata tengon lontana la calura con dolce frescura, qui me ne sto felice io dell'ombroso riparo e dei racemi di Libero, lieti di saporoso nettare. Non cento bocche dotte e voce di ferro saprebbero uguagliar di lodi i preziosi beni della campagna; vi scambieresti codeste gioie della città, piagate da veleno d'idra e dalla bocca di Cerbero». Ringrazio i colleghi latinisti Crescenzo Formicola e Giancarlo Abbamonte che hanno controllato la traduzione di questo e dei componimenti che seguono. 
La contrapposizione tra i ruris aurei bona e gli urbis gaudia è tema topico da Orazio in poi; il tema non è originale, ma sembrerebbe lecito dedurre dai versi di Anisio una implicita recusatio dell' invito a preferire la città rivoltogli da Garcilaso. Se i versi appena letti possono fare riferimento a un generico tema di conversazione tra un uomo che vive in corte tra armi e lettere e un umanista che delle lettere ha fatto scelta esclusiva, molto più confidenziali appaiono le espressioni del breve carme che si legge a c. $94 r$ :

Ad Charisylan seu Charsilassum
Seu te Minerva vertit in lapidem dea
sapientiae arduo obrigentem lumine,
seu praeda Charitum ob nobilem speciem animi
ac corporis tibi nomen istud indidit,
vel quod Pelasgi sic pilosum nominant
catumque sicco corde, denso pectore,
vel lassa nunquam beneficii est dandi domus tua,
nomen operis aestimamus inclytum
Charisyla amice Pieri sacris choris.

Dirò subito che non sono così sicuro dell'interpretazione data da Mele a suo tempo, né che io sia sicuro di poterne proporre una migliore, perché il latino dell'abate Anisio non sempre è agevole. Trovo però un po' fuori di chiave il giudizio di Mele $\mathrm{M}^{35}$, che giudicò di pessimo gusto la molteplice interpretatio nominis, dove invece a me pare sia più da cogliere una spia di intimità e di confidenza che rende possibile tra il serio e il faceto tratteggiare le doti spirituali e l'aspetto fisico dell'amico.

Provo a tradurre cercando di non allontanarmi dalla lettera del testo:

Sia che la dea Minerva ti abbia mutato nella pietra che si indurisce grazie al bagliore della più elevata sapienza, sia che per essere preda delle Grazie per il nobile aspetto dell'animo e del corpo ti abbia imposto codesto nome, o perché i Pelasgi così denominano un uomo peloso e reso accorto da un cuore arido [sereno, imperturbabile] e da un petto chiuso [impenetrabile], o perché il tuo lignaggio mai $\grave{e}$ stanco di concedere favori, stimiamo illustre la fama della tua opera, caro Garcilaso per le sacre danze di Piero [padre delle Muse].

La doppia variatio nominis indicata nel titolo (Charisyla seu Charsilassus) a rigore è sovrapponibile la prima ai vv. 3-4, in quanto formata su base greca con l'unione di charis (riferibile alle Grazie) e syle nell'accezione di 'preda', 'bottino', 'conquista'; la seconda ai vv. 4-5, tenendo conto che il ricorso alla metatesi charsis rimanda al sostantivo greco kàrsis/eos che significa 'tosatura', 'rasatura' accoppiato all'aggettivo latino lassus, che significa 'stanco', fa assumere al composto il significato 'stanco di rasatura' e quindi 'barbuto', 'peloso' (o Nemoroso $)^{36}$. Se invece si accettasse la proposta di Mele, che faceva riferimento

35. «Las poesías latinas de Garcilaso de la Vega», cit., p. 141.

36. Per la possibile relazione tra questa allusione di Anisio all'aspetto peloso e boscoso di Garcilaso e lo pseudonimo di Nemoroso si veda E. Fosalba, «Implicaciones teóricas del alegorismo autobiográfico en la égloga III de Garcilaso», Studia Aurea. Revista de Literatura Española y Teoría 
a un inesistente lemma greco làzios (in realtà làsios) nel significato di 'peloso', non si comprenderebbe la funzione di karsis nel composto che varrebbe 'peloso di tosatura', né perché mai Anisio così preciso nella scelta delle parole usasse l'aggettivo con raddoppiamento lassus e non lasus. Per i vv. 7-8 sembrerebbe sottintendersi una ulteriore variazione onomastica del tipo 'charis-lassus' o 'gracia-lassus' non compresa nel titolo, ma non riconducibile a rigore né a Charisyla né a Charsilassus. Più complicato decifrare quale possa essere il lapis obrigens, alluso dal nome, in cui il poeta è stato trasformato da Minerva "grazie al bagliore della più elevata sapienza", riferendo per enallage a sapientia l'agg. arduus, nel testo latino in concordanza con lumen. Occorre dire che Anisio qui gioca di fino, creando un fitto reticolo di allusioni convergente sul sostantivo silex (= selce). Muovendo dalla definizione di Isidoro di Siviglia (Ethymol. 16.3.1): "silex est durus lapis, eo quod exiliat ab eo ignis dictus ("la selce è così detta, perché da essa exiliat, balza fuori, il fuoco")», e passando per il Cornu copiae 1.1.10 di Niccolò Perotti ${ }^{37}$, testo di grande diffusione in ambito umanistico e sicuramente noto ad Anisio («et silex secundum quosdam, quod saxi genus est, quia silentem intra se ignem habeat qui attritu et percussu exciteturn) è possibile derivare l'idea che nei primi due versi la variatio nominis punti sulla forma charis-silex (= selce delle Grazie) nel senso che il bagliore folgorante di Minerva lo ha trasformato nella pietra che conserva dentro di sé il fuoco della sapienza capace di sprigionare scintille sotto l'azione delle Grazie. Non sono in grado di dire se Anisio accostasse l'etimologia di silex anche al greco sélas (= splendore, luce, lampo, fiamma) registrata nel più tardo Lexicon di Forcellini ${ }^{38}$ e quindi charis-sélas varrebbe 'fulgore delle Grazie', ma sempre per effetto del colpo di fulmine con il quale Minerva lo ha trasformato in silex che sprigiona scintille per attrito.

Non c'è dubbio che possa apparire eccessivamente confidenziale dare del 'barbuto' o del 'peloso' a un fascinoso hidalgo di Castiglia, di cattivo gusto non direi, se non altro perché a leggere bene potrebbe suonare persino come elogio, se e in quanto sembra cogliere la desenvoltura di un uomo di corte che esibisce i segni della sua virilità. Ma direi di più: «catumque sicco corde, denso pectore» potrebbe evidenziare, in quella accortezza o scaltrezza determinata «da un cuore arido (imperturbabile) e da un petto chiuso" (intendendo densus nel senso di 'impenetrabile, inaccessibile'), più che un atteggiamento di altezzosa aristocraticità, le doti di riservatezza che si richiedono all'uomo di corte cui il Viceré affidava delicati incarichi diplomatici. Se così non fosse, sembrerebbe immotivato il franco e chiaro elogio finale (uno dei primi se non il primo in assoluto) della musa di Garcilaso. Giacché in defintiva sembra che Anisio proprio questo volesse intendere: Garcilaso è un poeta eccellente, qualunque

Literaria del Renacimiento y Siglo de Oro, 3 (2009), 39-104, soprattutto 49 y 76.

37. Edizione a cura di J.-L. Charlet, Sassoferrato, Istituto internazionale di studi piceni, 1989, p. 22.

38. Totius Latinitatis lexicon, s. v. silex: «Silex est durus lapis eo quod exsiliat ignis, ab eo dictus.

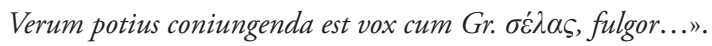


sia il modo in cui si voglia interpretare il suo nome. La scelta è ampia: charissilex/sélas, charis-syle, karsis-lassus, gracia-lassus.

6. Allargando per un po' lo sguardo all'insieme del liber di Giano Anisio pubblicato nel 1533 si può osservare come questo, a differenza dei due volumi stampati nel 1531 e nel 1532, quasi del tutto devoluti a fare bilancio e ricordo del passato e di amici e protettori in larga parte morti, sia pienamente immerso nella contemporaneità e come riveli un autore impegnato a dialogare con i nuovi venuti e accreditarsi come interlocutore affidabile, mostrando persino nei confronti di don Pedro de Toledo un atteggiamento di deferente cordialità, che lo spinge a suggerirgli un programma di governo per arginare il disordine politico del regno, in larga parte coincidente con le linee guida dell'azione politica che sarà dispiegata in processo di tempo dal Viceré. Ne estraggo i punti salienti:

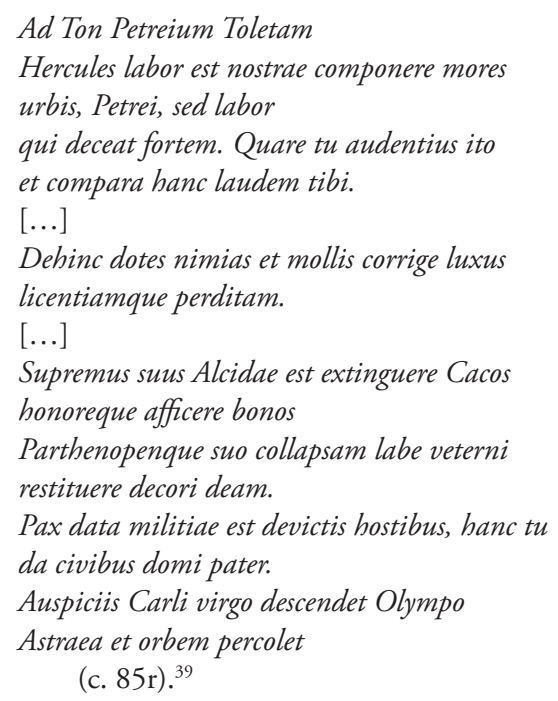

Anisio sembra interpretare il diffuso desiderio di riforma dei costumi e di consuetudini inveterate sulle quali allignavano le prepotenze del baronaggio e di corrotti funzionari dell'amministrazione, sicché, dopo aver auspicato uno stile di vita civile meno incline al lusso e perorato il risanamento urbanistico della capitale, invoca la restaurazione della giustizia, che saranno i due capisaldi del governo del toledano. Troppo precisi e numerosi i riferimenti alla strategia di governo del nuovo Viceré per ritenere che i versi siano esclusivo frutto di divinatio poetica e non piuttosto la cosciente e pronta adesione a un programma di lavoro, di cui Anisio si faceva disponibile divulgatore, presentandolo come

39. «È una fatica di Ercole, o Pietro, porre in ordine i costumi di Napoli, ma è fatica che si addice a un uomo coraggioso. Perciò osa di più e acquistati questa lode. [...] Quindi poni un freno alle doti eccessive e ai lussi smodati e alla dissolutezza sfrenata. [...] Il supremo lavoro proprio di Alcide è eliminare i Cachi e onorare i virtuosi e ricondurre Napoli divina collassata dalla decadenza della vetustà alla sua bellezza. In guerra è stata offerta la pace ai nemici sconfitti, questa stessa pace dona o padre ai cittadini in patria. Con i voti augurali di Carlo [V] la vergine Astrea tornerà dall'Olimpo e percorrerà di nuovo la terra». 
aspirazione della parte sana della società. È vero che chiunque ha facoltà di indirizzare versi alla propria autorità di governo, senza che questa autorità se ne accorga, ma a quei tempi un libro stampato non passava inosservato, tanto più che in entrambe le raccolte dei fratelli Anisio si leggono in assoluto i primi versi in lode del Viceré a lui dedicati da letterati napoletani (i quali anche in futuro non saranno mai generosi nei suoi confronti $)^{40}$. Quasi per accorciare le distanze, Giano ha disposto, poco prima di quello appena letto, un altro carme a don Pedro, nel quale, in forma ancora più estesa di quanto avesse fatto con Garcilaso, gli spiega perché preferisce la quiete della campagna di Domicilla alla vita della città ormai deserta di ogni virtù:

\author{
Ad Ton Petreium Toletam \\ [...] \\ Si quid opus tandem, Euboicam descendimus urbem \\ florentem Nolam, proximaque emporia. \\ Turba focos circum vernarum commoret: haec sunt \\ gaudia quae condit nectare simplicitas. \\ Simplicitas virtusque pedem hic posuere supremum \\ compulsae magnis urbibus ire procul. \\ Saepe mibi dulcis narrat Domicilla colonis \\ quae fuerit priscis vita, quod officium. \\ Commoda sed ruris complecti versibus esset \\ currere per magnum lyntribus Oceanum. \\ Rure datum nibil a diis est iucundius orbi, \\ foenus ubi uberius vitaque simplicior, \\ incompti lusus, incondita verba, cachinni, [...] \\ Haec tenuit reditum Remora praedulcis in urbem \\ Petrei, haec Siren mirificeque placet \\ (c. $83 \mathrm{r}){ }^{41}$
}

40. Si tenga conto che il poeta più organico alla corte di don Pedro, Luigi Tansillo, avrebbe atteso il 1551 per mandare alle stampe due smilze plaquettes poetiche (i Sonetti per la presa d'Africa e il [Capitolo per la liberazione di Venosa]): cf. T. R. Toscano, «Tra don Pedro e don García de Toledo: Luigi Tansillo cortegiano e precettore», in Encarnación Sánchez García (dir.), Rinascimento meridionale: Napoli e il viceré Pedro de Toledo (1532-1553), Napoli, Tullio Pironti, 2016, pp. 457-475.

41. «Se poi ho bisogno di qualcosa vado a Nola fiorente città di origini euboiche e ai mercati vicini. La servitù indugi vicino al camino: queste sono le gioie che la sincerità condisce del suo nettare. La semplicità e la virtù stabilirono qui l'ultima dimora una volta espulse dalle affollate città. Spesso la dolce Domicella mi racconta il modo di vivere e di lavorare degli antichi coloni. Ma dare pienamente conto in versi dei vantaggi della vita agreste sarebbe come navigare il vasto Oceano con una zattera. Gli dèi non hanno concesso al mondo niente che sia più piacevole della campagna, dove più copioso il guadagno e la vita più schietta, semplici i divertimenti, non troppo elaborato il discorrere, le risate. [...] Questa amabilissima Remora mi ha dissuaso dal ritorno nella città, e questa è la Sirena che mi diletta straordinariamente». Segnalo en passant il gusto dell'allusione peregrina che si cela dietro l'uso di Remora con l'iniziale maiuscola, variazione toponomastica per indicare Domicilla, da riconnettere al Remurinus ager lemmatizzato nella nota epitome del De verborum significatione di Pompeo Festo, realizzata da Paolo Diacono: "Remurinus ager dictus, quia possessus est a Remo: et habitatio Remi Remora: sed et locus in summo Aventino Remoria dicitur, ubi Remus de urbe condenda fuerit auspicatus» (P. Fest. 276 M.). Se però la tranquillità di Domicilla-Remora si contrappone al frastuono e al disordine della capitale, è 
Qui, accanto a Domicilla, viene evocata anche la vicina città di Nola, sufficiente a fornire tutto il necessario: una enclave territoriale in cui ancora è possibile vivere all'insegna della simplicitas: è l'insistenza su dei toponimi così particolari, che difficilmente si sarebbero citati se non identificabili per il destinatario, a indurre l'ipotesi che i versi siano la traccia di un breve soggiorno del Viceré e del suo seguito a Nola, in una fase cruciale peraltro della storia della città. Faccio una breve incursione nei versi di Luigi Tansillo, che molti anni dopo, nel 1551, ringraziando don Pedro per aver liberato Venosa, città in cui era nato da padre nolano, dal peso delle guarnigioni di soldati spagnoli, ricordò il debito di gratitudine contratto con lui per un beneficio accordato all'altra e più amata patria che era Nola:

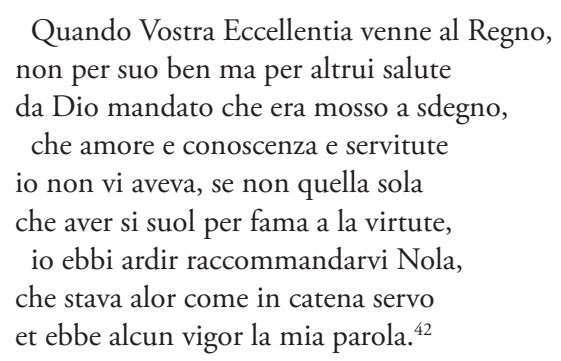

Si ritorna così agli inizi del viceregnato del Marchese di Villafranca: il poco più che ventenne Tansillo aveva perorato con felice esito la causa della sua città che stava alor come in catena servo: era infatti accaduto che don Pedro, tra i primi dossier da evadere trovò, per ordine di Carlo $\mathrm{V}^{43}$, la questione della demanializzazione della città già concessa a caro prezzo ai Nolani da Filiberto di Châlons principe di Orange il 29 luglio 1529, ma poi nuovamente infeudata a Francesca di Mombel principessa di Sulmona con diploma di Carlo V dato a Ratisbona il 21 giugno 1532; la città di Nola si oppose e l'Imperatore incaricò don Pedro di ripristinare il precedente atto del principe di Orange a patto che i nolani versassero alla principessa di Sulmona 10 mila scudi come adiutorio

probabile che Anisio faccia riferimento alla notizia riferita da Dionigi di Alicarnasso nel primo libro delle Antiquitates Romanae (cito dall'edizione, priva di segnatura e di cartulazione, stampata da Bernardino Celeri a Treviso nel 1480, in cui però il traduttore è erroneamente indicato come «Lappus Biragus Florentinus» (= Lapo da Castiglionchio il Giovane), trattandosi invece di Lampugnino Birago, allievo del Filelfo: sul quale cf. Massimo Miglio, Birago, Lampugnino, in Dizionario Biografico degli italiani, Roma, Istituto della Enciclopedia Italiana, 1968, t. 10), che a differenza di chi riteneva che Remora si dovesse localizzare sul colle Aventino, lo indica come "locus ad capiendam Urbem opportunus collis haud procul Tiberi, ad triginta ipse stadia Roma distans». Anisio poteva aver letto questa traduzione latina che ebbe larga circolazione, mentre è dubbio se potesse conoscere il luogo di Ennio (Annal. 77: «Certabant urbem Romam Remoramne uocarent»), in cui si fa riferimento alla disputa tra Romolo e Remo sul nome da attribuire alla città che intendevano fondare.

42. Luigi Tansillo, Capitoli giocosi e satirici, ed. C. Boccia e T. R. Toscano, Roma, Bulzoni Editore, col. «Europa delle Corti», 153, 2010, p. 345.

43. Lettera da Ratisbona del 2 settembre del 1532: cf. G. Vincenti, La contea di Nola dal sec. XIII al XVI, cit., p. 65. 
per acquistare la città di Boiano. Don Pedro avrebbe firmato il decreto che restituiva a Nola la condizione di città demaniale il 21 gennaio 1533 . Si tratta ovviamente di atti meramente amministrativi che non richiedevano di norma sopralluoghi, che comunque non si possono escludere. Le testimonianze incrociate di Anisio e Tansillo potrebbero conservare la traccia di un'escursione a Nola della corte vicereale, trattandosi per di più di città strategica nel sistema difensivo del regno. Resta che l'anno 1533 fu salutato dai nolani come anno primo della restituita libertà tanto che il vescovo del tempo fece incidere la memoria dell'evento nel bronzo della campana della rinnovata cattedrale ${ }^{44}$.

Chiusa questa digressione, osserverei infine che nel liber di Anisio del 1533 troviamo anche la prima menzione in assoluto di Luigi Tansillo, presentato non come poeta ma come giovane desideroso di apprendere il messaggio degli antichi e in particolare di conoscere la giusta interpretazione dell'aforisma socratico 'hoc unum scio, nihil scire':

\author{
Ad Elysium Tansillum \\ Quid sit, rogasti, Socratis verbum vetus: \\ quod maxime scio, illud unum nescio. \\ Audi quid ipse interpreter magnum senem \\ sensisse, mente et corde candide Elysi. \\ Deum esse sese scire certo certius \\ aiebat; at quid esset hoc nondum tamen \\ tot esse seclis cognitum mortalibus
} (c. $87 \mathrm{r}){ }^{45}$

Oltre ad aggregare alla sua conversazione poetica altro e sicuro amico di Garcilaso, Anisio aggiunge versi indirizzati a molte dame napoletane del tempo, e tra queste alcune che avrebbero incrociato la traiettoria napoletana di Garcilaso: ricordo almeno Caterina (Catalina) Sanseverino, cui l'anziano abate

44. Il fatto è narrato con molta enfasi da Gianstefano Remondini, Della nolana ecclesiastica storia, Napoli, Stamperia Simoniana, 1757, t. III, p. 212-214. L'eco dell'attesa delle città di Nola e di Lauro (quest'ultima facente parte della contea di Nola) di una adeguata ricompensa per gli atti valore compiuti a favore delle armi imperiali nel corso delle recenti invasioni francesi è anche in Cosma Anisio (Poemata 1533, cit., c. 66r), De Lauro et Nola: "Praemia debentur multis, sed praemia Laurus / Nolaque praecelso a Caesare digna petunt. / His siquidem amissis deploravere salute / Galli, tam turpis factaque deditio est, / Quippe fame non est homini pollentior hostis: I Tormenta haec vincit fulminaque ipsa Iovis» ("A molti sono dovuti dei premi, ma Lauro e Nola chiedono a Cesare [Carlo V] supremo premi adeguati: dal momento che, perduti questi [cioè Lauro e Nola] i Francesi disperarono della loro salvezza e fu stipulata una resa così vergognosa. Per l'uomo invero non esiste nemico più vigoroso della fame, la quale vince le punizioni e gli stessi fulmini di Giove»). Altro riferimento alla "liberazione" di Nola nel sonetto 151 di Luigi Tansillo a Giacomo Antonio Cesarini (cf. Rime, introduzione e testo a cura di T. R. Toscano, commento di E. Milburn e R. Pestarino, Roma, Bulzoni Editore, col. «Europa delle Corti» 154, 2011, t. II, pp. 552-553.

45. «Mi domandasti cosa voglia dire l'antico adagio di Socrate: ciò che so più a fondo, è quell'unica cosa che non so. Ascolta cosa io interpreto avere inteso il grande saggio, o Luigi candido di anima e cuore. Intendeva dire che egli sapeva con certezza che Dio esiste; ma cosa ciò fosse tuttavia non era ancora noto ai mortali in tanti secoli». 
dichiara un'ammirazione non priva di schietta nota di sensualità ${ }^{46}$, e i vari carmi dedicati a Maria di Cardona, cui Garcilaso avrebbe indirizzato il son. 24 (Illustre honor del nombre de Cardona), uno dei quali ne esalta la bellezza divina che la rende simile alle divintà:

\author{
De Merine Cardona \\ Si quis Iunonem Veneremque et Pallada nunquam \\ vidit praecipuas nobilitate deas \\ atque hic Cardonam vidit laeto ore Merinen, \\ conspexisse deas dixerit aetherias. \\ Sed quando est animus multo formosior ore \\ non levis hoc tantum cernere iudicii est \\ (c. $97 \mathrm{v}) .^{47}$
}

Il resto della storia non ci dice se Garcilaso abbia corrisposto all'amicizia di Giano Anisio: avrebbe potuto menzionare il vecchio umanista solo nell'ode latina ad Antonio Telesio (Uxore, natis, fratribus et solo / exul...), in cui affiorano nomi di amici e di sodali dell'epigonismo pontaniano. Inutile congetturare sull'esistenza di versi perduti di Garcilaso, che probabilmente non trovò stimolante l'interlocuzione con una poeta dedito esclusivamente alla musa latina e che nei confronti della poesia in volgare non cessò mai di esprimere le sue pesanti riserve ${ }^{48}$. Proprio l'epigramma di chiusura del liber del 1533, con la presa d'atto che ormai la scena letteraria è occupata dagli Orlandi e dalla lingua degenerata che ne narra le vicende, potrebbe dire che il poeta venuto di Spagna, giovane d'anni ma già maturo nell'arte, non poteva certo consentire a una liquidazione che avrebbe messo in discussione le sue stesse scelte:

46. A c. $91 r$, Ad Therinem Sanseveriniam: "O essem Zephyrus, cum terras Sirius urit, / spirantem ut mammis me caperes niveis. I O viola o essem rosa, odoro ut, dia Therine, I aut gremio aut istis perfruerer manibus.» ("Zefiro fossi io quando i campi dissecca Sirio, così che mi accogliessi come brezza tra le bianche mammelle. $\mathrm{O}$ viola $\mathrm{o}$ rosa io fossi sì da lambire, divina Caterina, o il tuo grembo olezzante o codeste mani»). Eugenio Mele, «Las poesías latinas de Garcilaso de la Vega», cit., p. 126, ricorda che nel testamento di Elena de Zuñiga, vedova di Garcilaso, si riconosce a Caterina Sanseverino un credito di 300 scudi prestati al poeta durante il suo soggiorno a Napoli.

47. «Se alcuno mai ha visto Giunone e Venere e Pallade, divinità eccellenti per nobiltà, e qui ha visto Maria di Cardona dal volto giocondo, avrebbe potuto dire di aver contemplato celesti dee. Ma poiché l'anima è molto più bella del volto, contemplare questa soltanto non è facoltà di superficiale discernimento».

48. D'altra parte nemmeno l'amicizia con il conterraneo Luigi Tansillo durò a lungo: nel 1538 Anisio stampò un violento epigramma in cui attaccava la produzione del più giovane poeta: «Quid Musa possit, Musa Apollinis soror, I non ista nata barbaris parentibus, / Tansille noscis, atque Anysium facis / parvi poetam lauream cinctum comas. / Hoc excitare dormientes est nepas» ("Di cosa sia capace la Musa, dico la Musa sorella di Apollo, non codesta (tua musa) nata da genitori bastardi, ignori, o Tansillo, e fai poco conto di Anisio poeta coronato di alloro. Questo vuol dire svegliare gli scorpioni in letargo»): Iani Anysii Epistolae de religione et epigrammata, Napoli, Sultzbach, 1538, c. $24 v$. Sul contesto e sulle motivazioni che furono alle origini di questa polemica cf. T. R. Toscano, Giano Anisio tra Nola e Napoli: amicizie, polemiche e dibattiti, in L'enigma di Galeazzo di Tarsia. Altri studi sulla letteratura a Napoli nel Cinquecento, Loffredo, Napoli 2004, pp. 79-102. 


\author{
Ad Librum \\ Quando non vivis blanditur fama poetis, \\ Et quis consciscat fata suprema sibi? \\ Posteritas nostros cognoscat ut aequa labores \\ Hos pluteus tristi vindicet a carie. \\ Cedendum interea Rholandis degenerique \\ Sermoni et secli fecibus historiae
}

Anisio si fa testimone dell'irresistibile avanzata della materia cavalleresca che soppianta la tematica storica, evidentemente per lui unico argomento degno di poesia: immagine viva del precoce e diffuso successo del Furioso di Ariosto a Napoli negli anni di Garcilaso ${ }^{50}$, e tale da gettare una luce di ambiguità sull'epitaffio che egli stesso aveva dettato in morte del poeta ferrarese scomparso pochi mesi prima ${ }^{51}$ :

\section{Epitaph(ium) Litavici Ariosti \\ Cum Litavico una occubuit Mars, Phoebus et ipsa \\ Gallorum heroum nobilis historia}

$$
\text { (c. } 97 \mathrm{v}) .^{52}
$$

A una prima lettura potrebbe sembrare un elogiativo compianto, e tale l'ho fin qui ritenuto, ma posto in relazione all'epigramma Ad librum, se colgo correttamente la polemica allusione alla marea montante degli Orlandi, sembrerebbe voler revocare in dubbio, morto l'Ariosto di cui implicitamente si riconosce il prestigio, la capacità della letteratura in volgare di sopravvivere continuando a coltivare, per adattare al nostro contesto una citazione da Bembo (Stanze 36, 7-8), «fole di romanzi e sogno et ombra, / che l'alme simplicette preme e 'ngombra». Perché in fondo Anisio neanche a Bembo era disposto a fare sconti; anzi, di lì a poco gli avrebbe imputato a dissipazione la dedizione eccessiva accordata al sermo tuscus chiamando in causa ancora una volta il «secolo ignorante»:

49. «E chi potrebbe decidere di decretarsi la morte dal momento che il successo non accarezza i poeti quando sono ancora in vita? Affinché la posterità, equanime, riconosca le nostre fatiche, uno scaffale le salvi dalla funesta corrosione. Nel frattempo la storia deve fare largo ai Rolandi e alla lingua degenerata e alla feccia del secolo».

50. Sulla fortuna dell'Orlando furioso a Napoli, largamente favorita dalla "corte" di Vittoria Colonna e, soprattutto, di Alfonso d'Avalos, marchese del Vasto, cf. T. R. Toscano, Letterati corti accademie. La letteratura a Napoli nella prima metà del Cinquecento, Napoli, Loffredo, 2000, pp. 104-108. Di particolare suggestione l'ipotesi di recente affacciata da Eugenia Fosalba «Más sobre la estancia de Garcilaso en Nápoles. Epigramas funerales a la muerte de Ariosto», in Encarnación Sánchez García (dir.), Rinascimento meridionale: Napoli e il viceré Pedro de Toledo (1532-1553), Napoli, Tullio Pironti, 2016, pp. 387-407) della attribuibilità a Garcilaso di un epicedio adespoto in morte di Ariosto, che si legge di seguito alle due odi latine di sicura attribuzione nel ms. XIII AA 63 (c. 64r) della Biblioteca Nazionale di Napoli.

51. Ariosto era morto il 6 settembre 1533, il liber di Giano è dello stesso anno.

52. «Insieme a Ludovico morirono Marte, Febo e persino la storia gloriosa degli eroi di Francia». 
O seclum inscitum: tot commentaria sermo tuscus habet, pauca et rancida Romuleus. Et nisi me Bembus sancto cohiberet amore illi ego nescio quid criminis intuleram: plus operae his nugis impendit nobilis heros quam tantum decuit forsitan ingenium. ${ }^{53}$

È possibile immaginare che il vecchio Anisio sia potuto apparire a Garcilaso un attardato laudator temporis acti chiuso nella difesa a oltranza della tradizione umanistica assediata dalla marea montante e inarrestabile della nuova letteratura in volgare, ma gli si può riconoscere almeno il merito di essere stato il primo a Napoli nel 1533 a consacrare l'inclytum nomen operis del poeta di Toledo.

53. «O secolo ignorante: la parlata toscana gode di tante trattazioni, contro le poche e stantie di cui dispone quella latina. E se Bembo non mi vincolasse di inviolabile amicizia, io non so di quale colpa lo accuserei: il nobil uomo dedica a codeste bagattelle un impegno maggiore di quanto forse sarebbe stato degno di ingegno così elevato": Iani Anysii Epistolae de religione, cit., c. $17 r$. La reprimenda a Bembo si legge in un componimento indirizzato Ad Flavium (probabilmente identificabile con il Giovanni Paolo Flavio, che nel 1535 aveva curato l'edizione del commento di Donato all'Eneide stampata a Napoli da Sultzbach, con dedica a don Luis de Toledo, figlio del Viceré, cui segue una lettera di Scipione Capece a Garcilaso de la Vega). Sempre a Bembo nel 1531 (Iani Anysii Varia poemata et satyrae, cit., c. 67v) aveva chiesto che insegnasse agli amici napoletani, "Camoenas qui faciles colunt, / curas edaces pallidasque / tristitias animo ut repellant» ("che coltivano Muse di facile accesso, a scacciare dall'animo passioni divoratrici e pallide tristezze»), in cui è evidente l'ironica presa di distanza dell'umanista nei confronti di rimatori di vena abbondante in tema di dolori e sospiri. 
